\title{
Effect of Asbestos on Anti-Tumor Immunity and Immunological Alteration in Patients with Malignant Mesothelioma
}

\author{
Yasumitsu Nishimura, Megumi Maeda, Naoko Kumagai-Takei, \\ Hidenori Matsuzaki, Suni Lee, Kazuya Fukuoka, Takashi Nakano, \\ Takumi Kishimoto and Takemi Otsuki
}

Additional information is available at the end of the chapter

http://dx.doi.org/10.5772/33138

\section{Introduction}

It is well known that malignant mesothelioma is caused by exposure to asbestos, which comprises a group of naturally occurring fibrous minerals. However, the mechanism by which asbestos causes malignant mesothelioma remains unclear. Many researchers have examined the effect of exposure to asbestos on the body. To date, it has been confirmed that asbestos can cause various forms of damage to cells, including cellular toxicity and mutagenicity, as well as produce reactive oxygen species (ROS) (Mossman \& Churg, 1998; Mossman et al., 1996; Sporn \& Roggli, 2004). The levels of oxidized pyrimidine and alkylated bases correlate with the period of occupational exposure to asbestos (Dusinska et al., 2004), and the increase in mutation frequency of lung DNA is caused by instillation of asbestos through the trachea (Topinka et al., 2004). All of these factors are thought to generate the tumorigenic effect of asbestos on mesothelial cells. However, the development of malignant mesothelioma caused by exposure to asbestos shows the noteworthy characteristics of this condition, which differ from those induced by other toxic materials. Malignant mesothelioma develops under a relatively low or medium dose of exposure to asbestos. A high dose of exposure to asbestos causes the development of pneumoconiosis, i.e., asbestosis rather than mesothelioma. Thus, the development of mesothelioma caused by exposure to asbestos cannot be explained only by a general rule regarding a dose-response relationship of toxic materials. In addition, it takes a long period of about forty years to develop malignant mesothelioma after exposure to asbestos. These findings suggest the existence of other factors related to the development of malignant mesothelioma that are modified by exposure to asbestos in the body, and which differ from the well-known 
tumorigenic effect of asbestos on mesothelial cells. One possible factor seems to be the effect of exposure to asbestos on anti-tumor immunity. In the body, the development of tumors is protected by anti-tumor immunity, composed of various kinds of cells including dendritic cells (DC), natural killer (NK) cells, helper T (Th) cells, cytotoxic T lymphocytes (CTLs), and so on. Exposure to asbestos might cause a suppressive effect on anti-tumor immunity in addition to the tumorigenic effect on mesothelial cells, and the combination of immunesuppressive and tumorigenic effects of asbestos might contribute to the development of malignant tumor (Fig. 1).
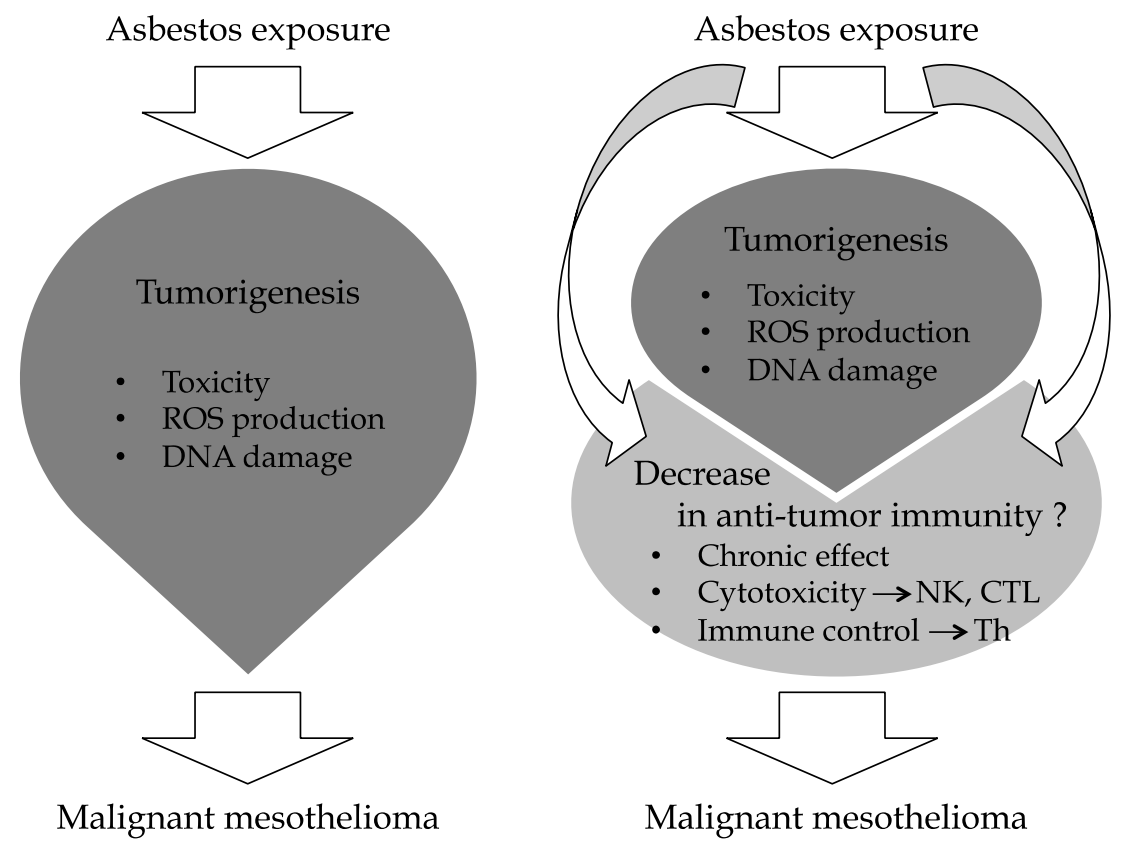

Figure 1. Hypothesis concerning the development of malignant mesothelioma caused by the immunological and tumorigenic effects of asbestos exposure. Many researchers have attempted to examine the tumorigenic effect of asbestos, thought to cause malignant mesothelioma (left). We propose the hypothesis that immune-suppressive effects, including the effect on NK- and T-cell functions, and the tumorigenic effects of asbestos exposure might contribute to the development of malignant mesothelioma (right).

The lung is not the only place where immune competent cells are able to meet asbestos fibers. Inhaled asbestos fibers reach the lung via the trachea, but they do not remain at that site. Those fibers translocate into the lung-draining lymph nodes over a long period. Dodson et al. examined the amount of asbestos in the lungs, lymph nodes, and plaque in a cohort of former shipyard workers, and reported that an analysis of asbestos in the lymph nodes confirmed accumulation in these sites (Dodson et al., 1991). In addition, asbestos fibers in the lymph nodes can translocate into the blood and may be observed in any tissue of the body, even in the brain where the accumulation of asbestos is low because of the blood- 
brain barrier (Miserocchi et al., 2008). Thus, immune competent cells have many opportunities to encounter asbestos fibers in the body, and the primary place where these cells meet asbestos seems to be the lung-draining lymph nodes (Fig. 2).

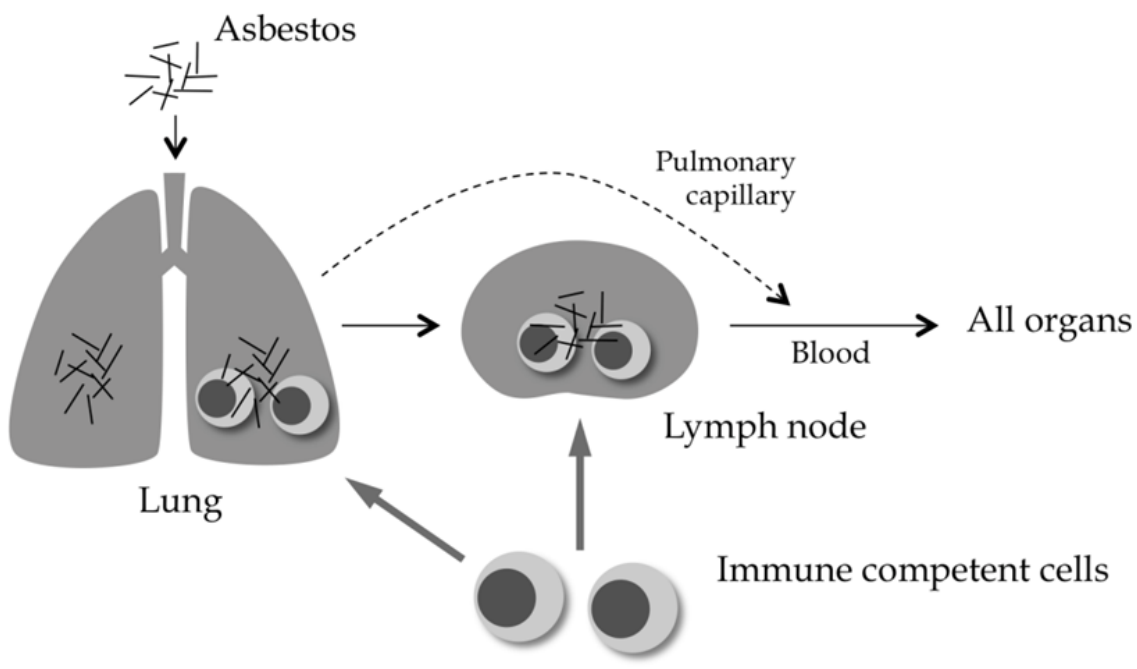

Figure 2. Places where immune competent cells encounter asbestos. Inhaled asbestos fibers reach the lung and translocate into the lung-draining lymph nodes. Therefore, the primary places where immune cells encounter asbestos seem to be the lung-draining lymph nodes.

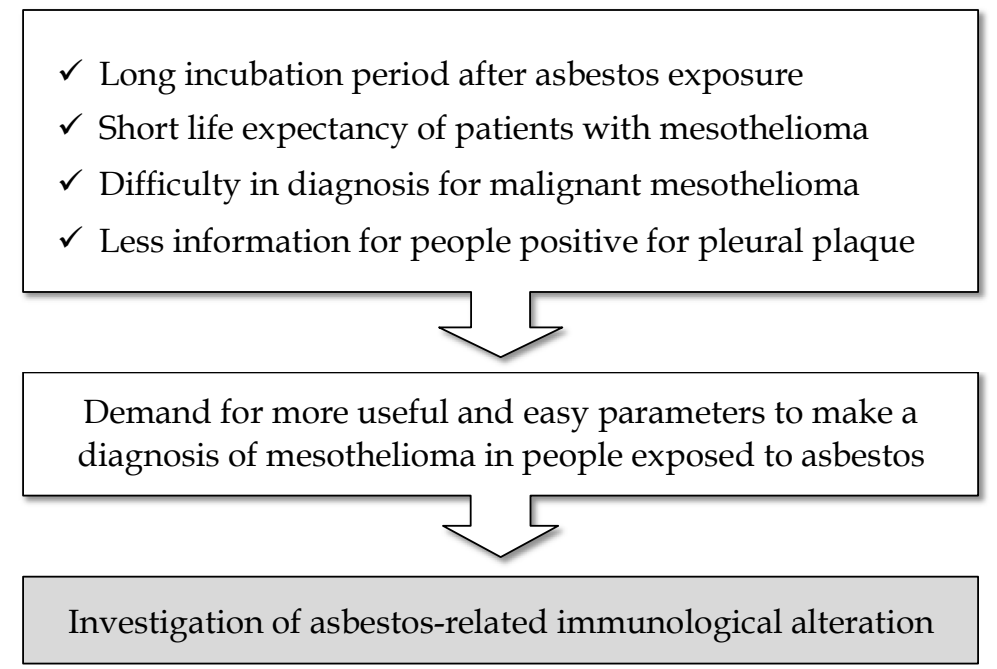

Figure 3. Present problems in malignant mesothelioma and the demand for a new parameter useful in making a diagnosis of malignant mesothelioma. Due to the problems shown in the top box, more useful and easy parameters to detect malignant mesothelioma in people exposed to asbestos are needed, to which our investigation of asbestos-related immunological alteration might contribute. 
The present diagnosis for malignant mesothelioma is based on X-ray and CT image analyses, as well as pathohistological analysis. However, diagnosis using these procedures is sometimes accompanied with a risk of radiation exposure or invasiveness, and results are not regarded equally among doctors because they have learned these analyses separately and it is not easy to master all of these methods. In addition, although it takes a long period to develop malignant mesothelioma after exposure to asbestos, the mean life expectancy of patients with malignant mesothelioma is short, and people exposed to asbestos need a safer analysis that can be used frequently in a year in order to detect malignant mesothelioma as early as possible. Recently, the use of products derived from mesothelioma cells for diagnosis has been proposed, including megakaryocyte potentiating factor (MPF) and mesothelin (Creaney et al., 2007; Onda et al., 2006). However, those products might not be observed in the blood that early because they will appear exactly after the development of mesothelioma, and it may take some time for those products to transfer from the pleural cavity into the blood stream. Many people exposed to asbestos worry about the development of malignant mesothelioma; however, there is little predictive information regarding the onset of mesothelioma. Therefore, there is a need to find a new parameter or method useful for the early diagnosis of malignant mesothelioma (Fig. 3). If some characteristic alteration of immune function is caused by exposure to asbestos that is also found in patients with malignant mesothelioma, which can be measured by checking lymphocytes or other cells in peripheral blood, the analysis for that alteration might contribute to the early detection of malignant mesothelioma.

On the basis of these ideas, we started to investigate the immunological effect of exposure to asbestos and immunological alteration in patients with malignant mesothelioma. In this chapter, we show the results obtained from these studies concerning the effect of asbestos on anti-tumor immunity, focusing on NK and Th cells, and discuss the immune-suppressive effect of asbestos and the possible application of our results for the early diagnosis of malignant mesothelioma.

\section{NK cells}

The role in cytotoxicity against target cells in anti-tumor immunity is played by two populations of cells, natural killer (NK) cells and cytotoxic T lymphocytes (CTLs). NK cells have a cytotoxicity for targets which they are ready to kill without prior stimulation, whereas this readiness is absent from the killing activity of CTLs and is induced by antigen stimulation. Therefore, although the cytotoxicity of NK cells has no antigen specificity, it is thought to contribute widely to the early deletion of unhealthy cells such as virus-infected cells or transformed cells. On the other hand, it takes time to induce the differentiation of naïve $\mathrm{CD}^{+} \mathrm{T}$ cells to CTLs, but they can recognize target cells precisely and injure them effectively. Imai et al. examined whether differences between individuals in regard to natural immunological host defense, i.e., NK cytotoxicity, can predict the future development of cancer. They reported that medium and high cytotoxic activity of peripheral blood lymphocytes is associated with reduced cancer risk, whereas low activity is associated with increased cancer risk, and these findings suggest a role for natural immunological host 
defense mechanisms against cancer (Imai et al., 2000). Therefore, we examined the effect of exposure to asbestos on cytotoxicity of NK cells and alteration in cytotoxicity of NK cells in patients with malignant mesothelioma.

\subsection{Mechanism of cytotoxicity in NK cells}

The mechanism of cytotoxicity in NK cells and CTLs can be separated into two parts. The role of one part is to recognize target cells, which is followed by transduction of the stimulation signal into the cytosol, while the other part acts to kill target cells. In the killing mechanism, both NK cells and CTLs use the common molecules perforin and granzymes. Perforin- and granzyme-induced apoptosis is the main pathway used by cytotoxic lymphocytes to eliminate virus-infected or transformed cells (Trapani \& Smyth, 2002). Perforin and granzymes are produced and accumulate in the cytotoxic granules of NK cells. Once NK cells are optimally stimulated, perforin and granzymes are released into the gap of the immune synapse by degranulation and act on target cells to induce apoptosis. Perforin is the protein that can disrupt the cellular membrane and create a pore in the membrane of the target cell. Granzyme is a family of structurally related serine proteases, which enters target cells through the pore made by perforin, and induces apoptosis of the target cells. The second pathway to kill targets is Fas-mediated apoptosis, induced by ligation of the Fas ligand (FasL) expressed on NK cells or CTLs with Fas on target cells. In addition to these two pathways, tumor-necrosis factor-related apoptosis-inducing ligand (TRAIL) is also known to control the growth and metastasis of tumors (Smyth et al., 2001; Takeda et al., 2001). These killing mechanisms are followed by recognition of target cells by NK cells. In contrast to $\mathrm{T}$ cell, which utilizes the $\mathrm{T}$ cell receptor (TCR) to recognize targets, NK cells utilize various kinds of receptors for target recognition. These receptors could be of either type: inhibitory or activating. In the next section, we explain the significance of the expression levels of NK cell receptors for cytolytic activity.

\subsection{NK cell receptors}

NK cells do not have clonal diversity like $\mathrm{T}$ cells, which include many repertoires, rearrangements, and somatic mutations of TCRs. However, NK cells can recognize various target cells using various kinds of receptors expressed on the cell surface of NK cells (Moretta, L. \& Moretta, A., 2004; Yokoyama \& Plougastel, 2003). Some NK cell receptors, the ligands of which are human leukocyte antigen (HLA) molecules, genetically differing among individuals and recognized by $\mathrm{T}$ cells with $\mathrm{T}$ cell receptor (TCR) to find abnormal cells, have the role of transducing an inhibitory signal. Those inhibitory receptors include a KIR family of receptors and heterodimer of NKG2A and CD94. The inhibitory signals derived from those receptors contribute to prohibition of cytotoxicity against normal self cells. In contrast, several other receptors transduce an activation signal after ligation with their respective ligands to induce cytotoxicity against abnormal target cells (Fig. 4). NKG2D is the best characterized activating receptor expressed on NK cells. NKG2D is a receptor belonging to the same group as NKG2A, NKG2 family, characterized by a lectin-like domain, but can transduce an activation signal unlike NKG2A. The signaling lymphocytic 
activation molecule (SLAM) family is another group of activating receptors expressed on NK cells. A representative receptor of the SLAM family is $2 \mathrm{~B} 4$, which induces cytotoxicity by stimulation with the natural ligand, CD48, or the antibody to 2B4 (Endt et al., 2007; Garni-Wagner et al., 1993; Valiante \& Trinchieri, 1993). Moreover, natural cytotoxicity receptors (NCRs) make a family of receptors that includes NKp46, NKp44 and NKp30, which play a major role in the NK-mediated killing of most tumor cells (Moretta, A. et al., 2001; Sivori et al., 1999). These activating receptors transduce the stimulation signal leading to the phosphorylation of c-Jun N-terminal kinases (JNKs) and extracellular signal-regulated kinase (ERK), which cause polarization of the microtubule organizing center (MTOC) and cytotoxic granules followed by release of perforin and granzymes, producing degranulation (Chen et al., 2006; Chen et al., 2007). Thus, the various kinds of receptors expressed on NK cells control induction of cytotoxicity for target cells, and alteration in expression of these receptors is thought to affect the strength of the stimulation signal to induce cytotoxicity of NK cells. In addition, if NK cells exposed to asbestos show some characteristic alteration in expression of NK cell receptors, this alteration might be used as a possible marker of asbestos exposure-related immune alteration. Therefore, we planned to study the effect of asbestos exposure on NK cells, focusing on the expression level of NK cell receptors, as well as investigate the cytotoxicity of NK cells.

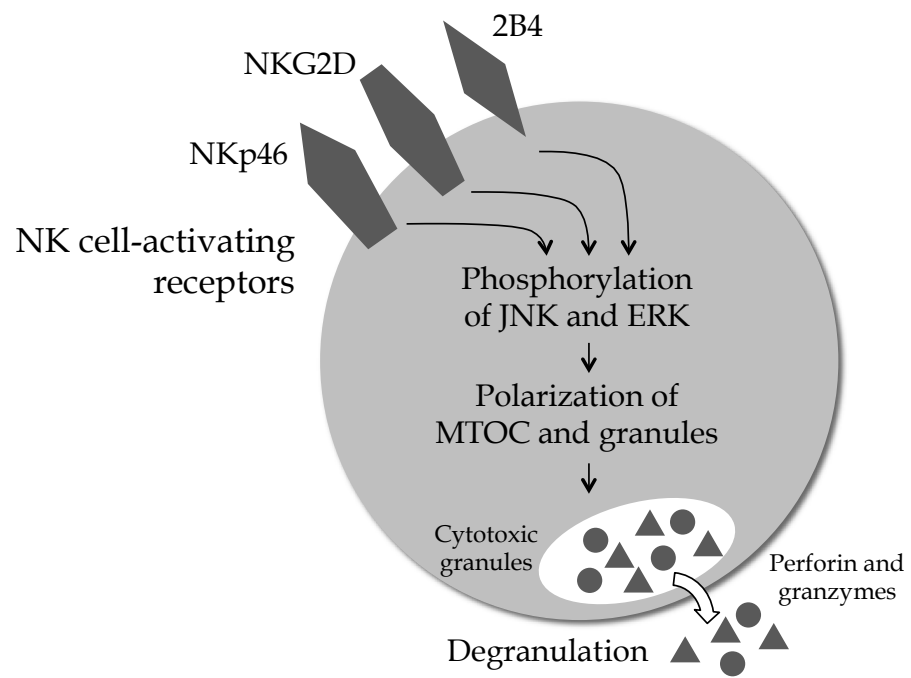

Figure 4. NK cell-activating receptors and the machinery of degranulation caused by stimulation with these receptors. The ligation of NK cell-activating receptors with their respective ligands induce phosphorylation of JNK and ERK, leading to polarization of the microtubule organizing center (MTOC) and granules, where perforin and granzymes are released and induce apoptosis of target cells.

\subsection{Cytotoxicity of human NK cell line cultured with asbestos for a long period}

We initially started by making the sub-line of an NK cell line by culturing cells exposed to asbestos for a long period. The human NK cell line of YT-A1 was kindly provided by Dr. 
Yodoi and was used for this experiment. Before starting the culture, the effect of exposure to chrysotile B (CB) on the survival and growth of YT-A1 cells was examined in order to avoid a high dose of exposure to $\mathrm{CB}$ which induces intense apoptosis and inhibition of cell growth. The apoptosis of YT-A1 was not induced upon exposure to $\mathrm{CB}$ at concentrations from 5 to $100 \mu \mathrm{g} / \mathrm{ml}$. However, cell growth was inhibited upon exposure to $\mathrm{CB}$ at a concentration greater than $50 \mu \mathrm{g} / \mathrm{ml}$, whereas it was not inhibited below $20 \mu \mathrm{g} / \mathrm{ml}$. Therefore, the dose of $\mathrm{CB}$ used was defined as $5 \mu \mathrm{g} / \mathrm{ml}$, representing the concentration at which YT-A1 cells were cultured with $\mathrm{CB}$, named YT-CB5, and the rest of cells was cultured in the original condition of media without $\mathrm{CB}$, named YT-Org. The cultures were exposed for over five months and then periodically examined for the effect of asbestos exposure on the cytotoxicity of NK cells. The cells were assayed for cytotoxicity against K562 cells and expression levels of various kinds of NK cell receptors and other molecules using flow cytometry. There were no differences in cytotoxicity or expression levels of cell-surface molecules between YT-Org and YT-CB5 for one month after exposure to CB. However, after around 5 months of such exposure, YT-CB5 showed a clear decrease in cytotoxicity compared with YT-Org. In addition, decreases in expression levels of NKG2D and 2B4, but not of NKp46, were also found in YT-CB5 (Nishimura et al., 2009b). The expression level of CD56 and CD16, NK-cell marker and low-affinity Fc receptor, respectively, altered slightly. NKG2A, which makes a suppressive receptor with CD94, did not increase. In addition, YT-CB5 showed almost a zero level of granzyme A and exhibited a significant decrease in perforin, but the degree of decrease of perforin was not large and YT-CB5 did not show a significant decrease in granzyme B. Although 2B4 is not related to cytotoxicity against $\mathrm{K} 562$ cells in contrast to NKG2D and NKp46, YT-CB5 also showed the decrease in cytotoxicity against P815 cells treated with antibodies to $2 \mathrm{~B} 4$, a cytotoxicity mediated by $2 \mathrm{~B} 4$. These results supported the supposition that the decrease in NKG2D and 2B4 might cause impairment in induction of cytotoxicity by reducing signal transduction downstream of those receptors. Therefore, the degranulation stimulated by antibodies to NKG2D and 2B4 was examined in YT-CB5 by flow cytometry, which can estimate degranulation by measuring an increase in cell-surface CD107a accompanied with secretion of cytotoxic granules. YT-CB5 showed decreases in degranulation stimulated via NKG2D and 2B4, as observed under stimulation with beadbound and plate-bound antibodies, respectively. In addition, YT-CB5 also showed a decrease in phosphorylation of ERK1/2 stimulated with K562 cells as well as antibodies to NKG2D, but not with antibodies to 2B4 (Nishimura et al., 2009a). Moreover, we examined cytotoxicity against K562 cells, expression levels of NKG2D, 2B4 and NKp46, and the phosphorylation level of ERK1/2 stimulated with their respective antibodies in peripheral blood (PB-) NK cells purified from the blood of healthy volunteers, and compared results among the volunteers. PB-NK cells with a high expression of NKG2D, derived from one individual, showed high cytotoxicity and high phosphorylation of ERK1/2, whereas both cytotoxicity and ERK phosphorylation were low in PB-NK cells with low NKG2D, derived from another individual. In a similar way, the relationship among the cell-surface level of NKp46, phosphorylation level of ERK1/2, and cytotoxicity was also investigated and confirmed in PB-NK cells of healthy volunteers. Thus, we demonstrated that exposure to 
asbestos caused an impairment in cytotoxicity of NK cells with decreases in NK cellactivating receptors. The decrease in NKG2D caused the low level of signal transduction, followed by a decrease in degranulation, in the asbestos-exposed subline of cells.

\subsection{Low cytotoxicity and low NKp46 level in NK cells of mesothelioma patients}

The result of the experiment using the human NK cell line described above suggested that inhaled asbestos might cause impairment in cytotoxicity with altered expression of NK cellactivating receptors, and that PB-NK cells in patients with malignant mesothelioma might show a similar impairment in cytotoxicity to YT-CB cells. Therefore, peripheral blood mononuclear cells (PBMCs) prepared from the blood of patients with mesothelioma were examined for cytotoxicity against K562 cells and expression levels of NKG2D, 2B4, and NKp46 in NK cells. The cytotoxicity of PBMCs against K562 in the tree different ratios of effector and targets was measured for each individual, and expressed as the percentage of specific lysis. To evaluate cytotoxicity per given number of NK cells, The number of NK cells in the cytotoxic reaction was calculated from the percentage of CD3-CD56 ${ }^{+}$NK cells in PBMCs and the number of PBMCs dispensed to each well of the culture plate for cytotoxic reaction. A linear regression line with the percentage of specific lysis and number of NK cells, in which the formula is [percentage of specific lysis] $=A$ [number of $N K$ cells] $+B$, was determined by using the three sets of percentage of specific lysis and number of NK cells for each individual. Finally, the percentages of specific lysis per 5000 NK cells from each individual were calculated from these regression lines and compared with those of healthy volunteers. Mesothelioma patients showed significantly lower cytotoxicity than healthy volunteers (Nishimura et al., 2009b). However, unlike YT-CB5, NK cells in patients with mesothelioma did not show a decrease in expression level of NKG2D or 2B4, whereas a decrease in NKp46 was observed in those NK cells. Thus, although NK cells in the peripheral blood of patients with malignant mesothelioma were not of the same character as YT-CB5, they also showed alteration in the expression of one of the NK-cell activating receptors, albeit a different one namely NKp46, with low expression of NKp46, and low cytotoxicity.

\subsection{Low cytotoxicity of NK cells with low NKp46 in the culture of PBMCs with asbestos}

As described above, NK cells in patients with malignant mesothelioma showed low cytotoxicity with low expression of NKp46, which was not found in YT-CB5. Therefore, to examine whether asbestos exposure causes such a decrease in NKp46 on NK cells, we performed a different experiment in which PBMCs from healthy volunteers were cultured in IL-2-supplemented media with chrysotile B at $5 \mu \mathrm{g} / \mathrm{ml}$, and after 7 days cells were harvested and examined for expression levels of NKG2D, 2B4, and NKp46 in CD3-CD56 ${ }^{+}$ NK cells by flow cytometry. IL-2 is a representative cytokine that induces proliferation and activation of NK cells and is commonly used to culture these cells. The results showed no decreases in cell-surface expression of NKG2D and 2B4 in NK cells derived from the culture 
with $\mathrm{CB}$, whereas the expression of NKp46 decreased in those NK cells, resembling NK cells of patients with malignant mesothelioma (Nishimura et al., 2009b). To determine whether such restrictive alteration in expression of NK cell-activating receptors is caused by exposure to other mineral fibers, PBMCs were cultured with glass wool and then examined for the expression level of NKp46 on NK cells. Glass wool is a kind of man-made mineral fiber and is used as a representative substitute for asbestos. However, unlike asbestos, exposure to glass wool did not cause a decrease in expression of NKp46. These results indicate that exposure to asbestos causes the characteristic abnormality in human primary NK cells, resulting in a decrease in NKp46 but not in NKG2D. As described above, this characteristic is similar to that of PB-NK cells in patients with malignant mesothelioma, suggesting the possible relationship between asbestos exposure-related suppression of NK cell function and development of malignant mesothelioma.

\section{3. $\mathrm{CD}^{+} \mathrm{T}$ cells}

What is the function of $\mathrm{CD}^{+} \mathrm{T}$ cells in regard to an appropriate immune response? $\mathrm{CD} 4^{+} \mathrm{T}$ cells, Th, contribute to various kinds of responses in innate and acquired immunity, including activation of NK cells, macrophages and dendritic cells (DC), as well as induction of antibody production and CTL development (Monney et al., 2002; Parker, 1993; Smith et al., 2004; Vivier et al., 2008). The production of various cytokines, including IL-2, IL-4, IL-5, IL-10, IL-17, IFN- $\gamma$, and TNF- $\alpha$, by Th cells is one of the reasons for the multiple contributions of those cells to immune function (Fig. 5).

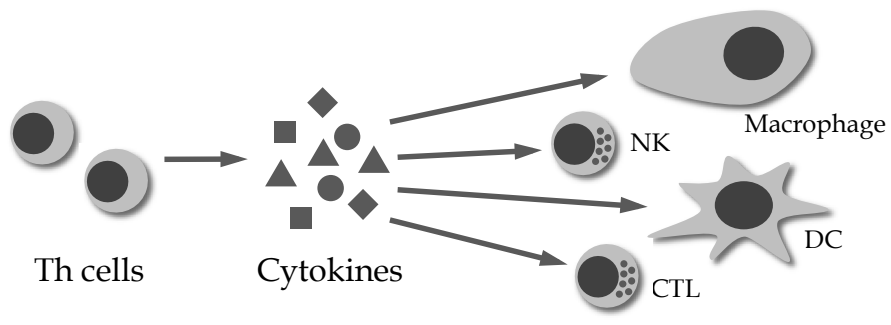

Cytokine-mediated control of functions

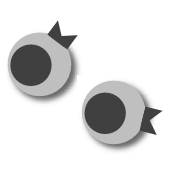

Th cells

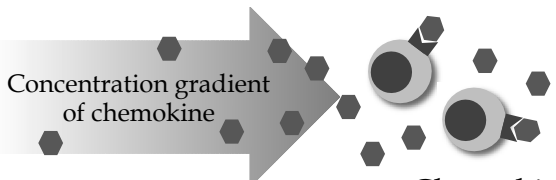

Chemokine-mediated control of migration

Figure 5. Cytokine-mediated control of immune functions and chemokine-mediated control of cell migration. The various kinds of cytokines produced by Th cells allow them to contribute widely to functions of immune competent cells (top). Concentration gradient of chemokines and expression of chemokine receptors play a key role in appropriate cell migration (bottom). 
In addition, cytokines produced by Th cells can be transported by the blood stream, which allows them to exert an influence on distant cells as well as adjacent cells. Moreover, immune competent cells can migrate widely throughout the body, and Th cells are no exception to this rule. Chemokines, a group of cytokines, play a large role in such cell migration. For example, a chemokine produced in the periphery creates a concentration gradient, by which cells having the receptor for that chemokine can be attracted. Th cell migration is also controlled in a similar manner, in which the expression of receptors for chemokines influences the migration. As described below, our study discovered that the immunological effect of asbestos exposure involved a characteristic alteration in cytokine production and expression of chemokine receptor in Th cells chronically exposed to asbestos, and that Th cells in patients with malignant mesothelioma exhibited characteristics similar to those of asbestos-exposed cells. Before elaborating on these findings, in the next section we will detail the cytokines produced by Th cells and the Th cell migration controlled by chemokines and chemokine receptors.

\subsection{Cytokines produced by Th cells and polarization to Th1, Th2, Treg and Th17 cells}

Antigen stimulation, made by a complex of MHC class II and antigen peptide, allows naïve Th cells to proliferate and produce cytokines. Th cells produce various kinds of cytokines, which can be classified according to the role they have in immune functions. TNF- $\alpha$ and IFN- $\gamma$ stimulate activation of NK cells and exert a promoting effect on the development of CTL, by which they support cell-mediated immunity for elimination of virus-infected or transformed/tumor cells. In contrast, IL-4 supports humoral immunity, in which it stimulates production of immunoglobulin, in particular IgE, by plasma cells differentiated from B cells, and is related to allergy and asthma. In addition, IFN- $\gamma$ and IL- 4 produced by Th cells show a mutual suppression in each other's production. As a result of such suppression, Th cells dominantly producing IFN- $\gamma$ show less production of IL-4 and vice versa. Therefore, such development of Th cells exhibits a dominant production of cytokinesis, called "polarization", and the cells showing dominant production of IFN- $\gamma$ and IL-4 are Th1 and Th2 cells, respectively (Liew, 2002). Moreover, part of the Th cells differentiate to regulatory $\mathrm{T}$ (Treg) cells, which dominantly produce IL-10 and TGF- $\beta$, immune-suppressive cytokines, and play a role in regulation of the immune response (Asseman et al., 1999; Dieckmann et al., 2002; Green et al., 2003; Jonuleit et al., 2002), although a part of Treg occurs naturally without antigen stimulation (Sakaguchi, 2005). It has also been reported that Th cells producing IL-17, called Th17, contribute to autoimmune diseases (Peck \& Mellins, 2009). Thus, several cytokines produced by Th cells and subpopulations of polarized Th cells show a characteristic role in immune functions. Therefore, immune status can be estimated by examining the production of these cytokines.

\subsection{Chemokine receptors expressed on Th cells}

Although immune competent cells are spread over the body, they must move to the place where they are needed according to their functions, and this also applies to each 
subpopulation of Th cells. Naïve T cells, both $\mathrm{CD} 4^{+}$and $\mathrm{CD} 8^{+}$cells, have to reach secondary lymphoid organs to check an antigen processed by antigen-presenting cells. In contrast, Th1 and Th2 cells, as effector cells, move to the periphery where they must encounter appropriate partners. Therefore, the expression of receptors for chemokines differs among the subpopulations of Th cells (Sallusto \& Lanzavecchia, 2000). Naïve T cells express the chemokine receptor CCR7, by which they acquire responsiveness to its ligands SLC and ELC and can move to secondary lymphoid organs. In contrast, Th1 and Th2 cells lose the expression of CCR7 and acquire the expression of CXCR3 and CCR5 or CCR3 and CCR4, respectively, although these expressions are a little flexible. CXCR3 is expressed on some NK cells, and CXCL10, the ligand of CXCR3, accumulates NK cells in tumors (Qin et al., 1998; Wendel et al., 2008). In addition, mice deficient for CXCL10 show impairment in T cell proliferation, IFN- $\gamma$ production, contact hyper-sensitivity, a representative Th1 response, and recruitment of $\mathrm{CD}^{+}$and $\mathrm{CD}^{+}$cells in the periphery (Dufour et al., 2002). On the other hand, CCR3 is expressed at high levels on eosinophils, basophils, and mast cells, the population of cells related to allergy and asthma (Gerber et al., 1997). Thus, the subpopulations of Th cells differ in the expression of chemokine receptors, which is related to their different roles in immune functions, and the assay for expression of chemokine receptors on Th cells allows us to obtain information regarding the balance of the Th1/Th2 response as well as cell migration.

\subsection{Impaired Th1 function of the human $\mathrm{T}$ cell line continuously exposed to asbestos}

As described above, many immune functions are under the control of Th cells. Therefore, we examined the effect of asbestos exposure on Th cells. At the beginning of this study, we prepared an in vitro T-cell model of long-term and low-level exposure to chrysotile asbestos using MT-2 cells, a human adult T-cell leukemia virus (HTLV)-1-immortalized human polyclonal cell line, resulting in six sublines exposed to asbestos. These sublines were established from the independent cultures of MT-2 cells with chrysotile asbestos. All of the sublines acquired resistance to asbestos-induced apoptosis after more than eight months of continuous exposure, and were named MT-2Rsts. Those six MT-2Rsts were used for DNA microarray analysis, compared with the original MT-2 cells, named MT-2Org. The analysis clarified statistically significant alterations in expression of 139 genes in MT-2Rsts, greater than twofold changes. To identify genes related to the suppression of anti-tumor immunity, the expression data were processed by the MetaCore Analytical Suite (http://www.genego.com; GeneGo, St. Joseph, MI) to search for deregulated networks and pathways. The results obtained from pathway and network analysis showed downregulation of IFN- $\gamma$ signalling and CXCR3 expression in MT-2Rsts. As mentioned above, both IFN $-\gamma$ and CXCR3 are related to Th1 cells. Therefore, we focused on Th1 functions of MT-2Rsts. All MT-2Rsts showed reduction of cell-surface expression of CXCR3, the mRNA level of which also decreased in MT-2Rsts, as assayed by real-time PCR. In addition, MT2Rsts showed a decrease in production of IFN- $\gamma$ compared to MT-2Org, as assayed by ELISA. Moreover, the production of CXCL10 also decreased in MT-2Rsts (Maeda et al., 
2010). These results indicate that continuous exposure of a human $T$ cell line to asbestos impaired Th1 function, leading to decreases in cell-surface expression of CXCR3 and production of IFN- $\gamma$ and CXCL10.

\subsection{Decreases in CXCR3 and IFN- $\gamma$ in primary $\mathrm{CD}^{+} \mathrm{T}$ cells exposed to asbestos}

Following the results obtained from the experiment using the MT-2 cell line, we examined the effect of asbestos exposure on human primary CD4 ${ }^{+} \mathrm{T}$ cells (Maeda et al., 2011). CD4+ $\mathrm{T}$ cells freshly isolated from PBMCs were stimulated with antibodies to CD3 and CD28 and cultured in IL-2-supplemented media for 3 days, and the activated CD4 ${ }^{+} \mathrm{T}$ cells were transferred into a new culture plate and cultured with IL-2 for a week. These polyclonally expanded $\mathrm{CD}^{+} \mathrm{T}$ cells were used for culture with chrysotile B asbestos. After 40 days of culture, cell surface CXCR3 expression decreased in a dose-dependent manner. In contrast, the expression of CCR5 varied among all healthy volunteers, and there were no significant changes after culture with chrysotile. In addition, we examined intracellular expression and the mRNA level of IFN- $\gamma$ in CD4 ${ }^{+}$T cells exposed to chrysotile B by flow cytometry and realtime PCR. The CD4 ${ }^{+} \mathrm{T}$ cells exposed to chrysotile B showed a decrease in IFN- $\gamma$ mRNA level, for which there was no significant difference, whereas IFN- $\gamma$ positive cells tended to be reduced in those asbestos-exposed cells. These results indicate that chronic exposure to asbestos caused the decrease in CXCR3 and IFN- $\gamma$ in $\mathrm{CD}^{+} \mathrm{T}$ cells, in accordance with results obtained from the experiment using the cell line.

\subsection{Decrease in $\mathrm{CD}^{+}{ }^{+} \mathrm{CXCR} 3^{+} \mathrm{T}$ cells in patients with mesothelioma}

Finally, we determined whether $\mathrm{CD}^{+} \mathrm{T}$ cells in asbestos-exposed patients showed the same impairment as shown by MT-2Rst sublines and in vitro asbestos-exposed primary CD4 ${ }^{+} \mathrm{T}$ cells (Maeda et al., 2011). Individuals positive for pleural plaque and patients with malignant mesothelioma were examined for CXCR3 expression and IFN- $\gamma$ production in peripheral blood $\mathrm{CD}^{+} \mathrm{T}$ cells. Both plaque-positive individuals and mesothelioma patients showed a significantly lower percentage of $\mathrm{CXCR}^{+}$cells in $\mathrm{CD}^{+} \mathrm{T}$ cells than healthy volunteers. In addition, the percentages of $\mathrm{CD}^{+} \mathrm{CXCR}^{+} \mathrm{T}$ cells in lymphocytes from the plaque and mesothelioma groups were also significantly lower than those of the healthy group, and the mesothelioma group showed the lowest percentage among the three groups. In contrast to CXCR3, the percentage of CCR5 $5^{+}$cells in $\mathrm{CD} 44^{+} \mathrm{T}$ cells and $\mathrm{CD} 4^{+} \mathrm{CCR} 5^{+} \mathrm{T}$ cells in lymphocytes was not low in the plaque and mesothelioma groups. To examine production of IFN- $\gamma$ by $\mathrm{CD}^{+} \mathrm{T}$ cells from plaque-positive individuals and mesothelioma patients, $\mathrm{CD} 4{ }^{+} \mathrm{T}$ cells were stimulated with antibodies to CD3 and CD28, and cells and culture supernatants were harvested and assayed for mRNA and secreted levels of IFN- $\gamma$. The $\mathrm{CD}^{+}{ }^{+} \mathrm{T}$ cells of mesothelioma patients showed a significantly lower mRNA level of IFN$\gamma$ than that of plaque-positive individuals or healthy volunteers, whereas the secreted level of IFN- $\gamma$ did not differ among the three groups. In addition, the concentration of CXCL10 in plasma tended to be higher for the plaque and mesothelioma groups than the healthy group, although there were no significant differences. CXCL10 can be produced by a variety of cells including endothelial cells, fibroblasts and monocytes near a cancerous lesion, to 
attract anti-tumor T cells (Luster \& Ravetch, 1987; Dufour et al., 2002; Homey et al., 2002). Moreover, the mesothelioma group showed a tendency for an inverse correlation between the percentage of $\mathrm{CD} 4{ }^{+} \mathrm{CXCR} 3^{+} \mathrm{T}$ cells and CXCL10 concentration, in comparison with the plaque and healthy groups. These results indicate that anti-tumor immune function in mesothelioma patients may be in the situation with less recruitment of Th1 cells using CXCR3, although the level of its ligand, CXCL10, is high.

\section{Conclusion and discussion}

We examined the effect of asbestos exposure on NK-cell and Th-cell functions using human NK- and T-cell lines continuously exposed to asbestos, primary cell cultures with asbestos, and analyses for peripheral blood NK and Th cells in plaque-positive individuals and patients with malignant mesothelioma. The results obtained from these studies indicate that asbestos exposure causes functional alterations in NK and Th cells, decreases in cytotoxicity and expression of NKG2D or NKp46, and decreases in production of IFN- $\gamma$ and expression of CXCR3, respectively. It is known that NK cell-activating receptors transduce a signal to induce phosphorylation of ERK and JNK, causing degranulation of cytotoxic granules. The results of our study also showed the relationship between the expression level of NKG2D or NKp46, ERK phosphorylation, and cytotoxicity using the NK cell line and peripheral blood NK cells from healthy individuals. Therefore, the asbestos-induced decrease in expression of NKG2D or NKp46 is thought to cause impairment of NK cell-mediated anti-tumor immunity. In addition, IFN- $\gamma$ is a key cytokine for the Th1 response and CXCR3 is one of the representative chemokine receptors expressed on Th1 cells. Therefore, the asbestos-induced decrease of IFN- $\gamma$ and CXCR3 in Th cells indicates the decrease of the Th1 response, which contributes to impairment of the immune response to tumors. These findings concerning NK and Th cells indicate that asbestos fibers have the potential to cause impairment of anti-tumor immunity. This is the first demonstration that asbestos exerts an immune suppressive effect, as well as a tumorigenic effect. The immune-suppressive effect of asbestos might contribute to development of malignant mesothelioma in people exposed to asbestos. As described in the introduction, asbestos is known to accumulate in the lung-draining lymph nodes, as well as the lungs, where NK and Th cells might be exposed to asbestos. Furthermore, the results of our studies indicate that several kinds of functional impairment in NK and Th cells observed in experiments of in vitro or ex vivo exposure to asbestos can also be observed in the cells of patients with malignant mesothelioma, although the results include some inconsistencies. NK cells in patients with mesothelioma showed the same decrease in NKp46 as NK cells in the PBMC culture with chrysotile asbestos, and Th cells in plaquepositive individuals and mesothelioma patients showed the same decrease in CXCR3 as the Th cell line and primary Th cells continuously cultured with chrysotile. In addition, it is noteworthy that there is also the consistency of parameters showing no alteration in expression between data from patients and cultures of primary cells. Those parameters are NKG2D and 2B4 for NK cells and CCR5 for Th cells, the altered expressions of which were not found in primary cells either exposed to asbestos or derived from patients with 
mesothelioma. These findings suggest that those characteristic functional alterations of NK and Th cells shown in patients with malignant mesothelioma might be caused by inhaled and accumulated asbestos in the body. Furthermore, they also suggest the possibility that decreases in NKp46 on NK cells and CXCR3 on Th cells might contribute to early diagnosis of malignant mesothelioma as markers to monitor asbestos-related immune suppression. Today, the diagnosis of malignant mesothelioma is dependent on X-ray and CT image analyses, as well as pathohistological analysis, but these procedures involve several problems such as difficulty in obtaining a consistent diagnosis and a risk of radiation exposure or invasiveness. In contrast, the drawing of blood necessary for analysis of immunological markers is safe and easy, and can be performed frequently during a year. Therefore, imaging and pathohistological analyses of malignant mesothelioma combined with immunological analysis for expression of NKp46 and CXCR3 might provide more valuable information for people who are exposed to asbestos and worry about the development of malignant mesothelioma. Further studies regarding the immunological effect of asbestos exposure will contribute to the effective diagnosis and therapy of malignant mesothelioma.

\section{Acknowledgment}

We thank Dr. Y. Yodoi for generously providing YT-Al cells, and Ms. Tamayo Hatayama, Satomi Hatada, Yoshiko Yamashita, Minako Kato, Tomoko Sueishi, Keiko Kimura, Misao Kuroki, Naomi Miyahara, and Shoko Yamamoto for their technical help. This study was supported by Special Coordination Funds for Promoting Science and Technology (H18-1-33-1), JSPS KAKENHI Grants (19790431, 18390186, 19659153, 19790411, 20890270, 20390178 and 22700933), The Takeda Science Foundation (Tokutei Kenkyu Josei I, 2008), Kawasaki Medical School Project Grants (18-209T, 19-407M, 19-603T, 19-205Y, 19-506, and 20-210O), and The Kawasaki Foundation for Medical Science and Medical Welfare (KYOIKU KENKYU JOSEI-2).

\section{Author details}

Yasumitsu Nishimura, Megumi Maeda, Naoko Kumagai-Takei, Hidenori Matsuzaki, Suni Lee and Takemi Otsuki Department of Hygiene, Kawasaki Medical School, Japan

Megumi Maeda Division of Bioscience, Department of Biofunctional Chemistry, Graduate School of Natural Science and Technology, Okayama University, Japan

Kazuya Fukuoka and Takashi Nakano

Department of Respiratory Medicine, Hyogo College of Medicine, Japan

Takumi Kishimoto

Okayama Rosai Hospital, Japan 


\section{References}

Asseman, C., Mauze, S., Leach, M.W., Coffman, R.L., \& Powrie, F. (1999). An essential role for interleukin 10 in the function of regulatory $\mathrm{T}$ cells that inhibit intestinal inflammation. The Journal of experimental medicine, Vol.190, No.7, pp.995-1004, 00221007

Chen, X., Allan, D.S., Krzewski, K., Ge, B., Kopcow, H., \& Strominger, J.L. (2006). CD28stimulated ERK2 phosphorylation is required for polarization of the microtubule organizing center and granules in YTS NK cells. Proc Natl Acad Sci U S A, Vol.103, No.27, pp.10346-10351, ISSN 0027-8424

Chen, X., Trivedi, P.P., Ge, B., Krzewski, K., \& Strominger, J.L. (2007). Many NK cell receptors activate ERK2 and JNK1 to trigger microtubule organizing center and granule polarization and cytotoxicity. Proc Natl Acad Sci U S A, Vol.104, No.15, pp.6329-6334, ISSN 0027-8424

Creaney, J., Van Bruggen, I., Hof, M., Segal, A., Musk, A.W., De Klerk, N., Horick, N., Skates, S.J., \& Robinson, B.W. (2007). Combined CA125 and mesothelin levels for the diagnosis of malignant mesothelioma. Chest, Vol.132, No.4, pp.1239-1246, 0012-3692

Dieckmann, D., Bruett, C.H., Ploettner, H., Lutz, M.B., \& Schuler, G. (2002). Human CD4(+)CD25(+) regulatory, contact-dependent $\mathrm{T}$ cells induce interleukin 10-producing, contact-independent type 1-like regulatory $\mathrm{T}$ cells [corrected]. The Journal of experimental medicine, Vol.196, No.2, pp.247-253, 0022-1007

Dodson, R.F., Williams, M.G., Jr., Corn, C.J., Brollo, A., \& Bianchi, C. (1991). A comparison of asbestos burden in lung parenchyma, lymph nodes, and plaques. Ann N Y Acad Sci, Vol.643, pp.53-60, ISSN 0077-8923

Dufour, J.H., Dziejman, M., Liu, M.T., Leung, J.H., Lane, T.E., \& Luster, A.D. (2002). IFNgamma-inducible protein 10 (IP-10; CXCL10)-deficient mice reveal a role for IP-10 in effector T cell generation and trafficking. J Immunol, Vol.168, No.7, pp.3195-3204, ISSN 0022-1767

Dusinska, M., Collins, A., Kazimirova, A., Barancokova, M., Harrington, V., Volkovova, K., Staruchova, M., Horska, A., Wsolova, L., Kocan, A., Petrik, J., Machata, M., Ratcliffe, B., \& Kyrtopoulos, S. (2004). Genotoxic effects of asbestos in humans. Mutat Res, Vol.553, No.1-2, pp.91-102, ISSN 0027-5107

Endt, J., Eissmann, P., Hoffmann, S.C., Meinke, S., Giese, T., \& Watzl, C. (2007). Modulation of 2B4 (CD244) activity and regulated SAP expression in human NK cells. Eur J Immunol, Vol.37, No.1, pp.193-198, ISSN 0014-2980

Fontenot, J.D., \& Rudensky, A.Y. (2005). A well adapted regulatory contrivance: regulatory $\mathrm{T}$ cell development and the forkhead family transcription factor Foxp3. Nat Immunol, Vol.6, No.4, pp.331-337, ISSN 1529-2908

Garni-Wagner, B.A., Purohit, A., Mathew, P.A., Bennett, M., \& Kumar, V. (1993). A novel function-associated molecule related to non-MHC-restricted cytotoxicity mediated by activated natural killer cells and T cells. J Immunol, Vol.151, No.1, pp.60-70, ISSN 00221767 
Gerber, B.O., Zanni, M.P., Uguccioni, M., Loetscher, M., Mackay, C.R., Pichler, W.J., Yawalkar, N., Baggiolini, M., \& Moser, B. (1997). Functional expression of the eotaxin receptor CCR3 in T lymphocytes co-localizing with eosinophils. Current biology : $C B$, Vol.7, No.11, pp.836-843, ISSN 0960-9822

Green, E.A., Gorelik, L., Mcgregor, C.M., Tran, E.H., \& Flavell, R.A. (2003). CD4+CD25+ T regulatory cells control anti-islet CD8 $+\mathrm{T}$ cells through TGF-beta-TGF-beta receptor interactions in type 1 diabetes. Proc Natl Acad Sci U S A, Vol.100, No.19, pp.1087810883, 0027-8424

Homey, B., Muller, A., \& Zlotnik, A. (2002). Chemokines: agents for the immunotherapy of cancer? Nat Rev Immunol, Vol.2, No.3, pp.175-184, 1474-1733

Imai, K., Matsuyama, S., Miyake, S., Suga, K., \& Nakachi, K. (2000). Natural cytotoxic activity of peripheral-blood lymphocytes and cancer incidence: an 11-year follow-up study of a general population. Lancet, Vol.356, No.9244, pp.1795-1799, ISSN 0140-6736

Jonuleit, H., Schmitt, E., Kakirman, H., Stassen, M., Knop, J., \& Enk, A.H. (2002). Infectious tolerance: human CD25(+) regulatory $\mathrm{T}$ cells convey suppressor activity to conventional CD4(+) T helper cells. The Journal of experimental medicine, Vol.196, No.2, pp.255-260, 0022-1007

Liew, F.Y. (2002). T(H)1 and T(H)2 cells: a historical perspective. Nature reviews Immunology, Vol.2, No.1, pp.55-60, ISSN 1474-1733

Luster, A.D., \& Ravetch, J.V. (1987). Biochemical characterization of a gamma interferoninducible cytokine (IP-10). The Journal of experimental medicine, Vol.166, No.4, pp.1084-1097, 0022-1007

Maeda, M., Nishimura, Y., Hayashi, H., Kumagai, N., Chen, Y., Murakami, S., Miura, Y., Hiratsuka, J.I., Kishimoto, T., \& Otsuki, T. (2011). Reduction of CXCR3 in an in vitro Model of Continuous Asbestos Exposure on a Human T-cell Line, MT-2. Am J Respir Cell Mol Biol, Vo.45, No.3, pp.470-479, ISSN 1535-4989

Maeda, M., Nishimura, Y., Hayashi, H., Kumagai, N., Chen, Y., Murakami, S., Miura, Y., Hiratsuka, J.I., Kishimoto, T., \& Otsuki, T. (2011). Decreased CXCR3 Expression in CD4+ T Cells Exposed to Asbestos or Derived from Asbestos-exposed Patients. Am J Respir Cell Mol Biol, Vo.45, No.4, pp.795-803, ISSN 1535-4989

Miserocchi, G., Sancini, G., Mantegazza, F., \& Chiappino, G. (2008). Translocation pathways for inhaled asbestos fibers. Environ Health, Vol.7, pp.4, ISSN 1476-069X (Electronic)

Monney, L., Sabatos, C.A., Gaglia, J.L., Ryu, A., Waldner, H., Chernova, T., Manning, S., Greenfield, E.A., Coyle, A.J., Sobel, R.A., Freeman, G.J., \& Kuchroo, V.K. (2002). Th1specific cell surface protein Tim-3 regulates macrophage activation and severity of an autoimmune disease. Nature, Vol.415, No.6871, pp.536-541, ISSN 0028-0836

Moretta, A., Bottino, C., Vitale, M., Pende, D., Cantoni, C., Mingari, M.C., Biassoni, R., \& Moretta, L. (2001). Activating receptors and coreceptors involved in human natural killer cell-mediated cytolysis. Annu Rev Immunol, Vol.19, pp.197-223, ISSN 0732-0582

Moretta, L., \& Moretta, A. (2004). Unravelling natural killer cell function: triggering and inhibitory human NK receptors. EMBO J, Vol.23, No.2, pp.255-259, ISSN 0261-4189

Mossman, B.T., \& Churg, A. (1998). Mechanisms in the pathogenesis of asbestosis and silicosis. Am J Respir Crit Care Med, Vol.157, No.5 Pt 1, pp.1666-1680, ISSN 1073-449X 
Mossman, B.T., Kamp, D.W., \& Weitzman, S.A. (1996). Mechanisms of carcinogenesis and clinical features of asbestos-associated cancers. Cancer Invest, Vol.14, No.5, pp.466-480, ISSN 0735-7907

Nishimura, Y., Maeda, M., Kumagai, N., Hayashi, H., Miura, Y., \& Otsuki, T. (2009a). Decrease in phosphorylation of ERK following decreased expression of NK cellactivating receptors in human NK cell line exposed to asbestos. Int I Immunopathol Pharmacol, Vol.22, No.4, pp.879-888, ISSN 0394-6320

Nishimura, Y., Miura, Y., Maeda, M., Kumagai, N., Murakami, S., Hayashi, H., Fukuoka, K., Nakano, T., \& Otsuki, T. (2009b). Impairment in cytotoxicity and expression of NK cellactivating receptors on human NK cells following exposure to asbestos fibers. Int J Immunopathol Pharmacol, Vol.22, No.3, pp.579-590, ISSN 0394-6320

Onda, M., Nagata, S., Ho, M., Bera, T.K., Hassan, R., Alexander, R.H., \& Pastan, I. (2006). Megakaryocyte potentiation factor cleaved from mesothelin precursor is a useful tumor marker in the serum of patients with mesothelioma. Clinical cancer research : an official journal of the American Association for Cancer Research, Vol.12, No.14 Pt 1, pp.42254231, 1078-0432

Parker, D.C. (1993). T cell-dependent B cell activation. Annu Rev Immunol, Vol.11, pp.331360, ISSN 0732-0582

Peck, A., \& Mellins, E.D. (2009). Breaking old paradigms: Th17 cells in autoimmune arthritis. Clin Immunol, Vol.132, No.3, pp.295-304, ISSN 1521-7035

Qin, S., Rottman, J.B., Myers, P., Kassam, N., Weinblatt, M., Loetscher, M., Koch, A.E., Moser, B., \& Mackay, C.R. (1998). The chemokine receptors CXCR3 and CCR5 mark subsets of $\mathrm{T}$ cells associated with certain inflammatory reactions. The Journal of clinical investigation, Vol.101, No.4, pp.746-754, ISSN 0021-9738

Sakaguchi, S. (2005). Naturally arising Foxp3-expressing CD25+CD4+ regulatory T cells in immunological tolerance to self and non-self. Nat Immunol, Vol.6, No.4, pp.345-352, ISSN 1529-2908

Sallusto, F., \& Lanzavecchia, A. (2000). Understanding dendritic cell and T-lymphocyte traffic through the analysis of chemokine receptor expression. Immunol Rev, Vol.177, pp.134-140, ISSN 0105-2896

Sivori, S., Pende, D., Bottino, C., Marcenaro, E., Pessino, A., Biassoni, R., Moretta, L., \& Moretta, A. (1999). NKp46 is the major triggering receptor involved in the natural cytotoxicity of fresh or cultured human NK cells. Correlation between surface density of NKp46 and natural cytotoxicity against autologous, allogeneic or xenogeneic target cells. Eur J Immunol, Vol.29, No.5, pp.1656-1666, ISSN 0014-2980

Smith, C.M., Wilson, N.S., Waithman, J., Villadangos, J.A., Carbone, F.R., Heath, W.R., \& Belz, G.T. (2004). Cognate CD4(+) T cell licensing of dendritic cells in CD8(+) T cell immunity. Nat Immunol, Vol.5, No.11, pp.1143-1148, ISSN 1529-2908

Smyth, M.J., Cretney, E., Takeda, K., Wiltrout, R.H., Sedger, L.M., Kayagaki, N., Yagita, H., \& Okumura, K. (2001). Tumor necrosis factor-related apoptosis-inducing ligand (TRAIL) contributes to interferon gamma-dependent natural killer cell protection from tumor metastasis. The Journal of experimental medicine, Vol.193, No.6, pp.661-670, ISSN 0022-1007 
Sporn, T.A., \& Roggli, V.L. (2004) Mesothelioma. In: Pathology of Asbestos-Associated Diseases, V.L. Roggli, T.D. Oury, T.A. Sporn, pp.104-168, Springer-Verlag, ISBN 978-1441918949, New York

Takeda, K., Hayakawa, Y., Smyth, M.J., Kayagaki, N., Yamaguchi, N., Kakuta, S., Iwakura, Y., Yagita, H., \& Okumura, K. (2001). Involvement of tumor necrosis factor-related apoptosis-inducing ligand in surveillance of tumor metastasis by liver natural killer cells. Nat Med, Vol.7, No.1, pp.94-100, ISSN 1078-8956

Topinka, J., Loli, P., Georgiadis, P., Dusinska, M., Hurbankova, M., Kovacikova, Z., Volkovova, K., Kazimirova, A., Barancokova, M., Tatrai, E., Oesterle, D., Wolff, T., \& Kyrtopoulos, S.A. (2004). Mutagenesis by asbestos in the lung of lambda-lacI transgenic rats. Mutat Res, Vol.553, No.1-2, pp.67-78, ISSN 0027-5107

Trapani, J.A., \& Smyth, M.J. (2002). Functional significance of the perforin/granzyme cell death pathway. Nat Rev Immunol, Vol.2, No.10, pp.735-747, ISSN 1474-1733

Valiante, N.M., \& Trinchieri, G. (1993). Identification of a novel signal transduction surface molecule on human cytotoxic lymphocytes. J Exp Med, Vol.178, No.4, pp.1397-1406, ISSN 0022-1007

Vivier, E., Tomasello, E., Baratin, M., Walzer, T., \& Ugolini, S. (2008). Functions of natural killer cells. Nat Immunol, Vol.9, No.5, pp.503-510, ISSN 1529-2916

Wendel, M., Galani, I.E., Suri-Payer, E., \& Cerwenka, A. (2008). Natural killer cell accumulation in tumors is dependent on IFN-gamma and CXCR3 ligands. Cancer Res, Vol.68, No.20, pp.8437-8445, ISSN 1538-7445

Yokoyama, W.M., \& Plougastel, B.F. (2003). Immune functions encoded by the natural killer gene complex. Nat Rev Immunol, Vol.3, No.4, pp.304-316, ISSN 1474-1733 